Paul Diepgen / Geschichte der Medizin 



\title{
GESCHICHTE DER MEDIZIN
}

\author{
DIE HISTORISCHE ENTWICKLUNG DER HEILKUNDE \\ UND DES ÄRZTLICHFN LEBENS
}

\author{
VON \\ PAUL DIEPGEN \\ PROIESSOR DR. MIED. DR. PHIL. DR. II.C. \\ MAINZ
}

II. BAND : I. HÄLFTE :

VON DER MEDIZIN DER AUFKLÄRUNG BIS ZUR BEGRÜNDUNG DER ZELLULARPATHOLOGIE (ca. 1740 - ca. 1858)

MIT 22 ABBILDUNGEN

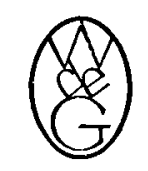

1951

IV A L TER DE GRUYTER \& CO. VORMALS G. J. GÖSCIIEN'SCHE VERLAGSHANDLUNG - J. GUTTENTAG, VERLAGSBUCHHANDLUNG · GLORG REIMER • KARL J. TRÜBNER • VEIT \& COMP. B ERLIN IV 35 
Alle Rechte, insbesondere das der Utbersetzung, vorbehalten.

Copyright 1951 by WALTER DE GRUYTER \& CO., vormals G. J. Göschen'sche Verlagshandlung - J. Guttentag, Verlagsbuchhandlung - Georg Reimer - Karl J. Trübner Veit \& Comp., Berlin W 35, Genthiner Straße 13. - Archiv-Nr. 515351

Printed in Germany. - Satz: Walter de Gruyter \& Co., Berlin W 35

Druck: Thormann u. Goetsch, Berlin SW 61 